CORRECTION

\title{
That gut feeling
}

\section{Anna Dart}

Nature Reviews Cancer 16, 756-757 (2016)

The page numbers for the reference for this Research Highlight were given incorrectly. The correct citation should be Daillère, R. et al. Enterococcus hirae and Barnesiella intestinihominis facilitate cyclophosphamide-induced therapeutic immunomodulatory effects. Immunity 45, 931-943 (2016). This has been corrected online. 